\title{
Revised Relativistic Hydrodynamical Model for Neutron-Star Binaries
}

\author{
G. J. Mathews \\ University of Notre Dame, Department of Physics, Notre Dame, Indiana 46556 \\ J. R. Wilson \\ University of California, Lawrence Livermore National Laboratory, Livermore, California 94550
}

(March 23, 2021)

We report on numerical results from a revised hydrodynamic simulation of binary neutron-star orbits near merger. We find that the correction recently identified by Flanagan significantly reduces but does not eliminate the neutron-star compression effect. Although results of the revised simulations show that the compression is reduced for a given total orbital angular momentum, the inner most stable circular orbit moves to closer separation distances. At these closer orbits significant compression and even collapse is still possible prior to merger for a sufficiently soft EOS. The reduced compression in the corrected simulation is consistent with other recent studies of rigid irrotational binaries in quasiequilibrium in which the compression effect is observed to be small. Another significant effect of this correction is that the derived binary orbital frequencies are now in closer agreement with post-Newtonian expectations.

PACS number(s): 97.80.Fk, 04.25.Dm, 04.40.Dg, 97.60.Jd

\section{INTRODUCTION}

In a recent paper E. Flanagan [1] has pointed out an inconsistency in the solution of the shift vector in our previously reported numerical hydrodynamic simulations [2 5] of binary neutron stars in quasiequilibrium orbits. In that paper it was suggested that this may be the source of the controversial relativistic hydrodynamic result that as the stars approach each other their interior density is observed to increase during numerical simulations.

In view of the controversial nature of the compression effect [6] and the fact that no other relativistic hydrodynamic treatments are yet capable of attacking this problem, it is of course essential to incorporate this correction into the previous hydrodynamic simulations to see if indeed this is the source of the observed compression effect.

In this short note we report of the first results of simulations in which this correction has been applied. We find that the correction does not eliminate the compression effect. It does, however, significantly diminish its magnitude at a given angular momentum. Perhaps more importantly, the correction causes the orbital frequency of the stars to increase such that the orbits are in closer agreement with post-Newtonian expectations. At the same time, however, this causes the inner most stable circular orbit (ISCO) to move to closer separation dis- tances and higher associated fluid velocities. The compression continues to scale with the magnitude $U$ of the spatial component four velocity as noted in [5]. Hence, by the time of inspiral and merger a significant compression effect is still possible. For a sufficiently soft equation of state, neutron stars in the mass range of observed neutron-star binaries still collapse to individual black holes.

\section{THE CORRECTION}

The correction pointed out by Flanagan concerns the the solution for the space-time components of the ADM $(3+1)$ metric

$$
d s^{2}=-\left(\alpha^{2}-\beta_{i} \beta^{i}\right) d t^{2}+2 \beta_{i} d x^{i} d t+\gamma_{i j} d x^{i} d x^{j},
$$

where the space-time components of the metric, $\beta^{i}$ are referred to as the shift vector.

We solve for the components of the shift vector by applying the ADM momentum constraint [7],

$$
D_{i}\left(K^{i j}-\gamma^{i j} K\right)=8 \pi S^{j} .
$$

Where $D_{j}$ is the three-space covariant derivative [7], and $S^{i}$ is the ADM three-momentum density.

In our approximation scheme, we impose maximalslicing $\left[\operatorname{Tr}\left(K^{i j}\right)=0\right]$ and demand that the spatial three metric $\gamma_{i j}$ be conformally-flat. Under these constraints, the second term on the left hand side of Eq. (2) vanishes and we have,

$$
D_{i} K^{i j}=8 \pi S^{j}
$$

Ultimately, we can reduce equation (3) to a Poisson-like equation for the shift vector,

$$
\nabla^{2} \beta^{i}=4 \pi \rho_{\beta}^{i}-\frac{\partial}{\partial x^{i}}\left(\frac{1}{3} \nabla \cdot \beta\right)
$$

The correction pointed out in [1] is in the derivation of the source density $\rho_{\beta}$. In [3] the contravariant spatial components of the four-momentum density were utilized in place of the contravariant three-momentum density. [This is valid for the covariant components but not for the contravariant components.] Hence, in [3] the source density was incorrectly written as, 


$$
\begin{aligned}
\rho_{\beta}^{i}= & 4 \alpha \phi^{4} S_{i}-4 \beta^{i} W^{2} \sigma \\
+ & \frac{1}{4 \pi \xi} \frac{\partial \xi}{\partial x^{j}}\left(\frac{\partial \beta^{i}}{\partial x^{j}}+\frac{\partial \beta^{j}}{\partial x^{i}}-\frac{2}{3} \delta_{i j} \frac{\partial \beta^{k}}{\partial x^{k}}\right),
\end{aligned}
$$

where $\sigma=\rho+\rho \epsilon+P$ is the inertial mass density, $\xi \equiv$ $\alpha / \phi^{6}, W \equiv \alpha U^{t}$, and

$$
S_{i}=\sigma W U_{i}
$$

The correct source should not have the second term on the right hand side. This is a spurious term which came from an erroneous identification of the contravariant momentum density with the ADM contravariant three-momentum density. The correct source density is,

$$
\rho_{\beta}^{i}=4 \alpha \phi^{4} S_{i}+\frac{1}{4 \pi \xi} \frac{\partial \xi}{\partial x^{j}}\left(\frac{\partial \beta^{i}}{\partial x^{j}}+\frac{\partial \beta^{j}}{\partial x^{i}}-\frac{2}{3} \delta_{i j} \frac{\partial \beta^{k}}{\partial x^{k}}\right),
$$

where $S_{i}$ is still given by Eq. (6).

This correction would have little effect on the final results if the spurious $4 \beta^{i} W^{2} \sigma$ term were small compared to the term containing $S_{i}$. That is not the case however. In the simulations of [3] it was observed that those two terms nearly cancelled leading to small values for the $\beta^{i}$. With this cancellation no longer in effect, the source for the shift vector equation is now a substantial quantity. This has several implications for the orbit dynamics which we now analyze.

\section{RESULTS}

\section{A. $\Gamma=2$ EOS}

As one way to identify the effect of this correction we have done calculations similar to the bench-mark calculation of [5], i.e. we have employed a simplistic $\Gamma=2$ polytropic equation of state (EOS), $P=K \rho^{\Gamma}$, where $K=1.8 \times 10^{5} \mathrm{erg} \mathrm{cm}^{3} \mathrm{~g}^{-2}$. This gives a maximum neutron-star mass of $1.82 M_{\odot}$. The gravitational mass of a single $m_{B}=1.625 M_{\odot}$ star in isolation is $1.51 M_{\odot}$ and the central density is $\rho_{c}=5.90 \times 10^{14} \mathrm{~g} \mathrm{~cm}^{-3}$. The compaction ratio for an isolated star with the same numerical grid resolution (in terms of Schwarzschild coordinates) is $m / R=0.14$. [Note that the central density for these stars these stars is slightly higher than quoted in [5] (for which $\rho_{c}=5.84 \times 10^{14} \mathrm{~g} \mathrm{~cm}^{-3}$ ). This is due to slight changes in the finite differencing of the present calculation.] The binary is taken to have a fixed angular momentum $J=2.5 \times 10^{11} \mathrm{~cm}^{2}\left(J / M_{G}^{2}=1.27\right.$ where $\left.\mathrm{M}_{G}=2 m_{G}\right)$. In quasiequilibrium circular orbit for this angular momentum, the proper (coordinate) separation distance between centers is $118(102) \mathrm{km}$ with a frequency consistent with post-Newtonian estimates. The stars relax to a nearly irrotational flow, and the central density only slightly increases by $0.8 \%$ to $5.95 \times 10^{14} \mathrm{~g} \mathrm{~cm}^{-3}$ as

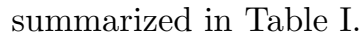

This very slight increase in central density is comparable to the numerical accuracy of the hydrodynamic calculation which we estimate to be $\sim \pm 0.5 \%$. It is also consistent with the very small amount of compression noted in simulations of purely irrotational $\Gamma=2$ stars by several groups 8 10] with a similar compaction ratio. For example, in [8] stars with a similar compaction ratio exhibit an increase in central density of $0.1 \%$ at this separation distance. In 9 changes in central density of order $0.1 \pm 0.5 \%$ are consistent with the numerical results; and in 10, stars with $m / R=0.14$ show a maximum central compression of about $2 \%$ at a coordinate separation distance of $d / R_{0}=1.6$ in their notation. A reasonable extrapolation of their figure 6 to our separation $\left(d / R_{0} \approx 4.5\right)$ suggests an increased central density consistent with our results.

\section{B. Realistic EOS}

In all of the above simulations [8 10, it has been noted that the compression effect dramatically increases for an EOS with an increased compaction ratio and closer orbits. Hence, in the remaining discussion we consider the "realistic" neutron star equation of state from 3, 3 , for which the compaction ratio is much higher $m / R \approx 0.25$. For this set of calculations, we not only compare with previous published results [3.4], but also quantify the effect of the correction on the location of the ISCO and the orbital frequency for the binary.

Consider first the somewhat soft EOS with a critical mass of $m_{c}=1.575$ and stars with a baryon mass of 1.548 $M_{\odot}$ corresponding to $\mathrm{M}_{G}=1.39 \mathrm{M}_{\odot} \rho_{c}=1.34 \times 10^{15} \mathrm{~g}$ $\mathrm{cm}^{-3}$ in isolation. These parameters roughly correspond 111] to one of the simulations in Table 2 of Ref. 迎. Table II compares differences between the previously derived

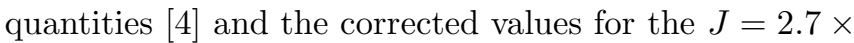
$10^{11} \mathrm{~cm}^{2}$ run. For the uncorrected runs, this $J$ was the last orbit before the stars collapsed.

Here, similar trends to those noted in the $\Gamma=2$ EOS are to be noted. The correction significantly stabilizes the stars for this $J$. However, the amount of compression $(\sim 3 \%)$ for this EOS is greater than for the simulation in Table

In Table III we show properties of corrected orbits as the stars approach the ISCO. For the uncorrected simulations the ISCO for these stars with a stiff EOS (i.e no collapse) occurred at $J \approx 2.0 \times 10^{11} \mathrm{~cm}^{2}$. In the corrected runs the orbital frequencies are larger for the same fixed angular momentum. This moves the ISCO in to closer distances and smaller $J$. Thus, although the compression effect is less for a given $J$, the stars reach higher velocities before the ISCO. This allows for significant compression ( $10 \%$ in this case) before the ISCO. Note, however, that these stars do not collapse as they had done in the uncorrected simulations. 
We did find, however, that collapse could occur if the stars were increased in mass from $m_{G}=1.39$ to 1.44 $\mathrm{M}_{\odot}\left(m_{B}=1.61 \mathrm{M}_{\odot}\right)$ for this simulation. The results from this run are given in Table IV. Collapse of the stars was observed to occur for very close orbits $(J=1.85 \times$ $10^{11} \mathrm{~cm}^{2}$ ) just before inspiral. The coordinate separation between stars was only 2.4 times the coordinate radii. At $J=1.8 \times 10^{11} \mathrm{~cm}^{2}$ the orbit still appeared stable though the stars wanted to collapse. In another calculation we used a softer EOS for which $m_{c}=1.54 \mathrm{M}_{\odot}$ and $m_{G}=$ $1.40, m_{b}=1.54 \mathrm{M}_{\odot}$. For this case, collapse occurred with $J=2.0 \times 10^{11} \mathrm{~cm}^{2}$.

Thus, collapse may still be a possibility albeit for stars close to the maximum mass of a soft EOS and for very close orbits. Such is soft EOS is a reasonable possibility. For example, collapse would always occur prior to inspiral for typical-mass neutron stars modeled with the EOS of Bethe and Brown 12 .

\section{Angular Momentum at the ISCO}

Another significant effect concerns the specific angular momentum $J / M_{G}^{2}$ as the stars approach the final orbits. In the previous results [13] the specific angular momentum at the ISCO was significantly higher $\left(J / M_{G}^{2} \approx 1.3\right)$ than that of a maximally rotating Kerr black hole. This would imply complicated dynamics during inspiral before the stars could merge. For the corrected results, $J / M_{G}^{2}=1.03$ for the last computed stable orbit of the sequence in Table III. For $J / M_{G}^{2}=0.99$ the orbit is unstable. Thus, we expect that when the stars begin to inspiral the specific angular momentum is very near unity, $J / M_{G}^{2} \approx 1.00$ and will become $\leq 1$ as the orbit plunges. Hence, the stars can immediately spiral inward to form a Kerr black hole near maximum rotation. This has important implications for the emergent gravity wave signal from the subsequent ringing.

\section{DISCUSSION}

Perhaps the most significant effect concerns the orbital frequency. The correction has caused the frequency to increase to be much closer to the expected post-Newtonian result (cf. Table II). It is easy to understand why this is so. In the hydrodynamic calculations, the orbital frequency $\omega$ is determined by minimizing the average coordinate three velocity, i.e.

$$
\langle V\rangle \propto\langle U-\beta-\omega \times r\rangle \approx 0
$$

The vector quantity $\beta$ is of opposite sign to $U$ and is much larger in the corrected simulations. This means that a larger value for $\omega$ is required to minimize the three velocity. This remains true even though the magnitude of $U$ for the binary slso slightly decreases.

A way of understanding the decrease in $U$ is to consider the balance between the relativistic analog of the centrifugal force and the gravitational force [3]. The $\beta$ part of the centrifugal force can be written as $S_{j} \partial \beta^{j} / \partial x^{i}$. This centrifugal force consists of two parts. One part scales as $U^{2}$ and one scales as $\beta U$. Since the $\beta$ term is now much larger the magnitude of $U$ required to balance the gravitational force becomes smaller.

Alternatively the reason for a decrease in $U$ while $\omega$ increases can be seen from the definition of angular momentum. In our simulations we constrain each orbit to a specific value for the covariant $\mathrm{z}$ component of the orbital angular momentum vector. In the corrected simulations the shift vector contributes significantly to the covariant angular momentum,

$$
J_{z}^{t o t} \equiv J=J_{z}^{S}+J_{z}^{\beta}
$$

where

$$
J_{z}^{S}=\int\left(x S_{y}-y S_{x}\right) \frac{\phi^{2}}{\alpha} d^{3} x
$$

and

$$
J_{z}^{\beta}=\int \sigma_{\beta}\left[y \beta^{x}-x \beta^{y}\right] d^{3} x
$$

where

$$
\sigma_{\beta}=\left(W^{2} \sigma-\rho \epsilon-P+\frac{1}{W}\right) \frac{\phi^{6}}{\alpha} .
$$

In the corrected equations $J_{z}^{\beta}$ is a significant fraction of the total angular momentum $(\underset{\sim}{<} 1 / 2$, see Table II $)$. Thus, the corresponding momentum density contributions $\left(J_{z}^{S}\right)$ for a given total angular momentum is less. This constrains $U$ to be smaller in equation 10 .

In 3-5] it was noted that the increase in central density scales with the four velocity for the binary as $\rho_{c} \propto U^{4}$. It is note worthy that the $U^{4}$ scaling with a similar proportionality constant still applies to the corrected results as shown in Table [V]. The main difference, however, is that the values of $U$ are lower after the correction. Hence, the compression is less for a given total $J$.

\section{CONCLUSION}

In summary, we have applied the revision recently pointed out by E. Flanagan [1] into a hydrodynamic simulation of binary neutron stars near the ISCO. The effects of this correction have been analyzed. Although the magnitude of the compression effect is reduced for a given fixed total angular momentum, some compression effect remains. The scaling of the compression with the 
spatial components of the four velocity $U$ remains the same. The main difference is that $U$ is smaller for a fixed angular momentum. This is at least in part because a significant fraction of the covariant angular momentum now arises from the corrected shift vector term.

Another outcome is that the corrected orbital angular frequencies are higher than the uncorrected results. This brings the anticipated orbital and gravity wave frequences into closer alignment with post-Newtonian estimates. Another consequence of the higher orbital angular frequencies is that the ISCO for the binary moves in to closer separation distances, higher velocities, and higher orbital angular momenta than previously estimated. This increases the compression such that collapse to two black holes is still possible before inspiral, although only for a softer EOS and/or higher mass. The possibility that the EOS is this soft is not yet ruled out [12,14 18 .

Although we note here that some compression remains in the revised hydrodynamics, its magnitude is considerably reduced. The question remains then as to whether the remaining effect is real or an artifact of the uncertainties [5,8] introduced by the conformally flat condition on the metric. The resolution of this question, however, will require simulations in which the full Einstein dynamics are included in the orbits. We are currently developing a perturbation expansion to examine this question.

Work at University of Notre Dame supported by NSF grant PHY-97-22086. Work at the Lawrence Livermore National Laboratory performed in part under the auspices of the U. S. Department of Energy under contract W-7405-ENG-48 and NSF grant PHY-9401636.

[1] E. Flanagan, Phys. Rev. Lett., 82, 1354 (1999).

[2] J.R. Wilson and G.J. Mathews, Phys. Rev. Lett. 75, 4161 (1995).

[3] J.R. Wilson, G.J. Mathews, and P. Marronetti, Phys. Rev. D54, 1317 (1996).

[4] G. J. Mathews and J. R. Wilson, Astrophys. J. 482, 929 (1997).

[5] G. J. Mathews, P. Marronetti and J. R. Wilson, Phys. Rev. D58, 043003 (1998).

[6] D. Lai, Phys. Rev. Lett. 76, 4878 (1996; A. G. Wiseman, Phys. Rev. Lett. 79, 1189 (1997); M. Shibata, Prog. Theor. Phys. 96, 317 (1996); M. Shibata, K. Taniguchi, and T. Nakamura, Prog. Theor. Phys. 128, 295 (1997); K. Taniguchi and T. Nakamura, Prog. Theor. Phys. 96, 693 (1996); D. Lai and A. G. Wiseman, Phys. Rev. D54, 3958 (1996); W. Ogawaguchi and Y. Kojima, Prog. Theor. Phys. 96, 901 (1996). J. C. Lombardi, F. A. Rasio, and S. L. Shapiro, Phys. Rev. D56, 3416 (1997); P. Brady and S. Hughes, Phys. Rev. Lett. 79, 1186 (1997); E. Flanagan, Phys. Rev. D58, 124030 (1998); K. Thorne, Phys.
Rev. D58 (1998) 124031; M. Shibata, T. W. Baumgarte, and S. L. Shapiro, Phys. Rev. D58 (1998) 023002.

[7] J. W. York, Jr., in Sources of Gravitational Radiation, ed. L . Smarr (Cambridge; Cambridge Univ. Press) p. 83 (1979); C. R. Evans, PhD. Thesis, Univ. Texas (1985).

[8] S. Bonazzola, E. Gourgoulhon, J.-A. Marck, in Proc. 19th Texas Symposium on Relativistic Astrophysics and Cosmology, held in Paris, France, Dec. 14-18, 1998. Eds.: J. Paul, T. Montmerle, and E. Aubourg (CEA Saclay), 1999, in press; Phys. Rev.Lett. 82 (1999) 892-895

[9] P. Marronetti, G. J. Mathews, and J. R. Wilson, Phys. Rev. D60 (1999) 087301.

[10] Uryu, K. \& Eriguchi, Y. 1999, PRD submitted, grqc/9906102

[11] The equation of state utilized in the present simulation gives a gravitational mass which is differs by $<1 \%$ from the calculations of 迎. This difference is insignificant for purposes the present comparison.

[12] H. A. Bethe and G. E. Brown, Astrophys. J., 445, L129, (1995).

[13] D. M. Eardley, E. W. Hinchman, (unpublished preprint) gr-qc/9601019.

[14] Lattimer, J. 1998, in Neutron Stars and Pulsars, N. Shibazaki et al., eds., Front. Science Ser. No. 24, Universal Academic Press, inc. Tokyo), pp. 103-110.

[15] S. E. Thorsett and D. Chakrabarty, Astrophys. J., 512:288-299, 1999.

[16] C. J. Pethick, A. Akmal, V. R. Pandharipande, D. G. Ravenhall, in Proc. 19th Texas Symposium on Relativistic Astrophysics and Cosmology, held in Paris, France, Dec. 14-18, 1998. Eds.: J. Paul, T. Montmerle, and E. Aubourg (CEA Saclay), 1999, in press.

[17] C. Schaab, C. and M. K. Weigel, MNRAS, 308, 497, (1999), astro-ph/9904211

[18] J. C. Salmonson, J. R. Wilson and G. J. Mathews, 1999, submitted to ApJ 
TABLE I. Comparison between corrected and uncorrected results for $\mathrm{m}_{B}=1.625 \mathrm{M}_{\odot}\left(m_{G}=1.51 \mathrm{M}_{\odot}\right.$ in isolation $)$ stars in a binary with $J=2.5 \times 10^{11} \mathrm{~cm}^{2}$ and a $\Gamma=2$ EOS.

\begin{tabular}{cccc}
\hline \hline Quantity & $\begin{array}{c}\text { Isolated } \\
\text { Star }\end{array}$ & $\begin{array}{c}\text { Corrected } \\
\text { Binary }\end{array}$ & $\begin{array}{c}\text { Uncorrected } \\
\text { Binary }\end{array}$ \\
\hline$d_{p}(\mathrm{~km})$ & $\infty$ & 118 & 138 \\
$\rho_{c}\left(10^{14} \mathrm{~g} \mathrm{~cm}^{-3}\right)$ & 5.90 & 5.95 & 6.68 \\
$\left(\delta \rho / \rho_{0}\right)$ & 0. & 0.008 & 0.14 \\
& & & \\
\hline \hline
\end{tabular}

TABLE II. Comparison between corrected and uncorrected results (from [4]) for $m_{B}=1.548 \mathrm{M}_{\odot}\left(m_{G}=1.39\right.$ $\mathrm{M}_{\odot}$ in isolation) stars in a binary with $J=2.7 \times 10^{11} \mathrm{~cm}^{2}$ and a realistic EOS.

\begin{tabular}{ccc}
\hline \hline Quantity & Corrected & Uncorrected \\
\hline$d_{p}(\mathrm{~km})$ & 61 & 68 \\
$\rho_{c}\left(10^{15} \mathrm{~g} \mathrm{~cm}^{-3}\right)$ & 1.38 & 1.98 \\
$\left(\delta \rho / \rho_{0}\right)$ & 0.03 & 0.51 \\
$\omega\left(\mathrm{rad} \mathrm{sec}^{-1}\right)$ & 1330 & 732 \\
$\left(\omega / \omega_{P N}\right)$ & 1.01 & 0.66 \\
$U^{2}=W^{2}-1$ & 0.0091 & 0.034 \\
$J_{z}^{S}\left(10^{11} \mathrm{~cm}^{2}\right)$ & 1.4 & 2.7 \\
$J_{z}^{\beta}\left(10^{11}\right) \mathrm{cm}^{2}$ & 1.3 & - \\
$\alpha_{\min }$ & 0.55 & .44 \\
\hline \hline
\end{tabular}

TABLE III. Summary of corrected results for $\mathrm{m}_{B}=1.548$ $\mathrm{M}_{\odot}\left(m_{G}=1.39 \mathrm{M}_{\odot}\right.$ in isolation $)$ binary stars with a realistic EOS.

\begin{tabular}{ccccc}
\hline \hline$J\left(10^{11} \mathrm{~cm}^{2}\right) \omega\left(\mathrm{rad} \mathrm{sec}^{-1}\right)$ & $d_{p}(\mathrm{~km})$ & $\rho_{c}\left(10^{15} \mathrm{~g} \mathrm{~cm}^{-3}\right)$ & $U^{2}$ \\
& & & & \\
$\infty$ & 0.0 & $\infty$ & 1.34 & 0.0 \\
3.0 & 1025 & 72.0 & 1.37 & .0080 \\
2.8 & 1190 & 65.6 & 1.375 & .0085 \\
2.6 & 1460 & 56.2 & 1.38 & .0097 \\
2.4 & 1660 & 51.4 & 1.40 & .0115 \\
2.2 & 2110 & 43.8 & 1.43 & .0145 \\
2.1 & 2425 & 42.0 & 1.44 & .0165 \\
2.0 & 2530 & 41.2 & 1.45 & .0185 \\
1.9 & 2750 & 39.0 & 1.455 & .020 \\
1.8 & 3000 & 35.0 & 1.46 & .023 \\
1.7 & 3450 & 34.0 & 1.47 & .0245 \\
1.65 & 4200 & 29.2 & 1.50 & .0335 \\
1.6 & $($ Inspiral $)$ & & & \\
\hline
\end{tabular}

TABLE IV. Summary of corrected results for $\mathrm{m}_{B}=1.61$ $\mathrm{M}_{\odot}\left(m_{G}=1.44 \mathrm{M}_{\odot}\right.$ in isolation $)$ binary stars with a realistic EOS.

\begin{tabular}{ccccc}
\hline \hline$J\left(10^{11} \mathrm{~cm}^{2}\right)$ & $\left.\omega(\mathrm{rad} \mathrm{sec})^{-1}\right)$ & $d_{p}(\mathrm{~km}) \rho_{c}\left(10^{15} \mathrm{~g} \mathrm{~cm}^{-3}\right)$ & $U^{2}$ \\
\hline & & & & \\
$\infty$ & & & & \\
3.0 & 1710 & 56.4 & 1.38 & 0.0 \\
2.6 & 1800 & 52.0 & 1.55 & .013 \\
2.4 & 2100 & 47.2 & 1.57 & .015 \\
2.2 & 2400 & 44.2 & 1.60 & .020 \\
2.0 & 3000 & 37.2 & 1.64 & .030 \\
1.9 & 3600 & 29.8 & 1.72 & .040 \\
1.85 (Collapsing) & 4500 & 23.6 & 4.05 & .070 \\
& & & & \\
\hline \hline
\end{tabular}

